\title{
Carnets
}

Revue électronique d'études françaises de l'APEF

Deuxième série - 4 | 2015

Regards sur Camus

\section{Actualité d'Albert Camus sur la scène espagnole}

\section{Rafael Ruiz Álvarez}

\section{(2) OpenEdition}

\section{Journals}

Édition électronique

URL : http://journals.openedition.org/carnets/1575

DOI : 10.4000/carnets. 1575

ISSN : 1646-7698

Éditeur

APEF

\section{Référence électronique}

Rafael Ruiz Álvarez, «Actualité d'Albert Camus sur la scène espagnole », Carnets [En ligne], Deuxième série - 4 | 2015, mis en ligne le 22 septembre 2016, consulté le 04 mai 2019. URL : http:// journals.openedition.org/carnets/1575; DOI : 10.4000/carnets.1575

Ce document a été généré automatiquement le 4 mai 2019.

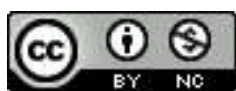

Carnets est mis à disposition selon les termes de la licence Creative Commons - Atribution - Pas d'utilisation commerciale 4.0 International. 


\title{
Actualité d'Albert Camus sur la scène espagnole
}

\author{
Rafael Ruiz Álvarez
}

1 Caligula est un personnage de l'histoire de Rome qui a surtout éveillé des émotions négatives. Souvent qualifié de pervers, de fou, de tyran anxieux d'éprouver l'abîme du pouvoir, il n'est pas moins vrai qu'il incarne aussi la séduction d'une personnalité qui s'impose aux autres de par sa quête arbitraire et subjective de la liberté1. Caligula ne laisse aucun interlocuteur indifférent. Le cinéma, le théâtre et la littérature nous offrent encore de manière fréquente des portraits de l'empereur soulignant tantôt sa force tantôt sa faiblesse ainsi que son ambigüité, sa cruauté et sa sensibilité, son intelligence et son mépris de l'amour, des dieux, des hommes et de soi-même ${ }^{2}$. Sa trace dépasse les limites temporelles et spatiales parvenant à envahir les plateaux du monde entier depuis que Camus décida d'en faire le héros d'une de ses pièces de théâtre. C'est ainsi qu'en Espagne le personnage est convoqué depuis cinquante ans de manière régulière sur les scènes les plus réputées du pays. En effet c'était déjà en 1963 que José Tamayo l'exhiba sur le plateau splendide du théâtre romain de Mérida ${ }^{3}$. Depuis lors nous avons eu au moins une quinzaine de propositions différentes de ce grand classique dont la plupart font recours au même référent littéraire déjà cité : Albert Camus. L'explication se trouve dans le fait que le prix Nobel français a assuré à travers des phrases lapidaires et de profondes réflexions l'immortalité non pas seulement de son personnage et de son époque mais aussi de toute une conception philosophique et sociale sur l'idée de l'être humain pleine de vigueur et de validité aujourd'hui. Le texte de Camus donc exerce une espèce d'hypnose inspirant le respect le plus profond à ceux qui l'abordent et à ceux qui décident de le transposer sur scène.

2 C'est bien en hommage du célèbre auteur que nous avons choisi d'analyser trois adaptations de son texte en espagnol restant unies par une fidélité complète au même mais proposant des modalités différentes par rapport à la conception de l'espace scénique et des langages de la mise en scène ${ }^{4}$. Ces adaptations sont celle de 1971, mise en scène de Jorge Azpilicueta, le rôle principal interprété par José María Rodero ; celle de 2009, sous 
l'empreinte de Santiago Sánchez et l'interprétation de Sandro Cordero ; et celle de 2012, orchestrée par Joaquín Vida, le rôle de Caligula étant accordé à Javier Collados 5 .

Nous avons décidé de prendre comme point de départ l'adaptation d'Azpilicueta, qui reprend à son tour celle que Tamayo avait déjà réalisée avec Rodero. Cette foi-ci la mise en scène a une valeur supplémentaire pour nous: le fait de constituer le premier document audiovisuel de la pièce dont nous disposons. Cela veut dire que cette version utilise la télévision comme support et comme moyen de transmission du spectacle théâtral au spectateur. Un spectateur passif auquel on offre tous les détails que l'on veut souligner. TVE avait conçu une émission consacrée au théâtre - Estudio 1 - dans le but d'offrir au public un répertoire de pièces nationales et internationales déjà célèbres afin de satisfaire la demande intellectuelle d'un spectateur cultivé. Dans ce sens nous considérons que l'adaptation d'Azpilicueta possède une conception artistique impeccable, pleine de nuances à l'heure d'interpréter le texte camusien. Le metteur en scène soigne à la perfection tous les langages scéniques et filmiques en les agençant suivant une orientation - l'époque et la censure l'exigeait - historiciste et traditionnelle, mais sans mépriser l'opportunité que ces nouveaux moyens techniques offraient à l'heure d'aborder les différents sujets clés de la pièce de Camus ainsi que l'interprétation des différents rôles, notamment celui de Caligula. Cette adaptation sera par conséquent le point de départ de notre analyse car elle constitue de plus le référent successif des metteurs en scène, des acteurs et des professionnels de la représentation théâtrale.

4 La deuxième adaptation qui fera l'objet de nos réflexions a été conçue par la troupe l'Om Imprebìs. Cette compagnie dédie ses productions à mettre en rapport le théâtre avec des situations sociales de dénonciation. Elle maintient par ailleurs une politique de décentralisation de la figure de l'acteur principal en faveur d'une conception globale du spectacle où sont soulignés les différents langages de la mise en scène. Leur objectif repose sur l'idée de réfléchir sur les pièces qu'ils offrent dans une intention pédagogique consciencieusement élaborée, toucher un public qui n'est pas familiarisé avec le théâtre et qui méconnaît les pièces des grands auteurs ${ }^{6}$.

5 La troisième adaptation, la plus récente, celle de la coopérative Cosmoarte, naît de l'initiative d'un groupe d'acteurs à vocation d'entreprise théâtrale. Ils ont voulu faire du théâtre, du bon théâtre de texte et d'auteur, garantissant un accueil favorable du public. La combinaison Camus-Caligula-Vida-Collados a très bien marché7. Les responsables du projet assuraient donc leur réussite, même en étant conscients des risques d'ordre historique qu'ils ont assumés octroyant à Caligula personnage un côté plus humain et plus sensible que celui de l'histoire, capable d'éveiller chez les autres personnages et surtout chez le spectateur une réaction logique plus actuelle.

Une fois présentées les trois adaptations espagnoles du texte de Camus auxquelles nous allons nous rapporter il s'impose d'établir les axes de réflexion sur lesquels nous allons organiser notre analyse. Il est évident que la partie discursive, le langage verbal de la pièce camusienne ne fera pas l'objet primordial de notre étude car il faut penser que les textes espagnols qui en dérivent sont plutôt des traductions du français à l'espagnol. Cela veut dire que ce qui est vraiment intéressant pour nos réflexions - n'oublions pas que notre titre souligne l'actualité de Camus en Espagne - relève surtout de la manière dont ces adaptations réinterprètent le texte source à partir des langages de la représentation théâtrale. Les didascalies externes de la pièce de Camus pourront nous servir énormément à ce propos ainsi que ces autres à caractère interne qui s'en dégagent et qui 
porteront sur trois volets: les langages de la mise en scène, l'empreinte de l'acteur principal dans le rôle de Caligula et la part du spectateur d'aujourd'hui.

\section{Des langages de la mise en scène ${ }^{8}$}

7 Pour commencer nous allons mentionner l'espace référentiel que Camus propose dans son texte et qui s'articule en deux lieux : le palais de Caligula et la maison de Cherea. Ce dernier pour le deuxième acte seulement, mais impliquant une rupture des unités de lieu et de temps et l'éloignant des règles classiques appliquées aux tragédies d'ordre historique. Peu de détails de l'auteur d'ailleurs permettant son transfert à l'espace scénique à l'exception des colonnes, des statues ou des gradins cités dans son texte ainsi que des éléments accessoires qui configurent une scénographie et des outils propres à l'époque à laquelle on se rapporte : quelques éléments pour s'asseoir - sans préciser leur caractéristiques-, un miroir, une table, un gong, un maillet, un sifflet...

8 L'adaptation d'Azpilicueta se présente dans ce sens au spectateur comme un travail qui mêle les éléments théâtraux aux éléments cinématographiques grâce au support de la télévision'. Il s'agit donc plutôt de théâtre filmé ou de captation sans public dans ce caslà. Le metteur en scène - à la rigueur le réalisateur aussi - peut tirer profit de cette situation et par conséquent utiliser des moyens techniques plus suggestifs pour un spectateur habitué au petit écran. La première chose que celui-ci voit c'est un générique suivi d'une voix over récitant des fragments de l'essai L'homme révolté, de Camus. Ce préambule met l'accent sur la destinée tragique de l'être humain et sert à situer le spectateur dans un contexte qui le prévient du contenu philosophique de la pièce qu'il va voir et de sa portée intellectuelle. Il sera par la suite plongé dans une atmosphère propre à l'époque romaine qui reproduit en espace reconstitué ce que Camus proposait dans son texte. Or Azpilicueta se servira du pouvoir de la caméra pour mieux marquer les scènes qui ont lieu dans le palais de Caligula face à celles de la maison de Cherea ou celles qui se passent à l'extérieur de la demeure de l'empereur. Les images offertes désignent très clairement ces espaces différents. Le spectateur connaît par conséquent qu'il y a eu un déplacement physique de l'action linéaire ce qui est de plus remarqué par les fondus au noir qui séparent chaque acte.

Il y a d'autres aspects des langages scéniques qui contribuent à manifester l'originalité de l'adaptation d'Azpilicueta ou au contraire sa fidélité au contexte historique sans que Camus révèle un intérêt excessif à ce propos. En ce qui concerne l'apparence externe des acteurs par exemple on peut souligner que les costumes sont en correspondance avec l'époque dont il s'agit. Le noir et blanc de la télévision ne rend pas suffisamment claires les tonalités et les nuances qu'ils puissent offrir, mais on apprécie cependant le travail de recherche des professionnels qui ont opté par un souci d'authenticité et de réalisme à cet égard.

Bien différente se montre le langage rythmico-musical. En effet, la musique est essentielle dans cette adaptation. Elle y est très présente et y joue le rôle assigné à ce langage pour le cinéma: transmettre des émotions chez les spectateurs, suggérer des sentiments des personnages, remarquer des aspects de leur personnalité, créer une ambiance déterminée de tension, de romance, d'effroi... Toutes les grandes scènes de la pièce - moments de folie et de solitude de Caligula, jeux grotesques de l'empereur face aux sénateurs, assassinat de Caesonia, mise à mort de Caligula - sont renforcés par ce moyen, véritable bande sonore over ${ }^{10}$. 
11 Il faut également souligner l'excellent travail de la caméra qui s'éloigne de ce que le théâtre est en réalité et qui est propre au cinéma mais qui accorde ici une valeur extraordinaire à l'interprétation et à la dimension tragique de la pièce telle qu'Azpilicueta la conçoit. Elle accorde à la scène une ambiance tragique qui repose sur la maladie et la souffrance du héros. On peut remarquer à ce propos l'apparition sur scène de Caligula, très frappante, où le metteur en scène fait appel à l'espace désigné par le hors-scène - le jardin de la pièce camusienne où l'on dit avoir vu Caligula d'un « air égaré, sale, cheveux pleins d'eaux » (Camus, $1958: 25)$. Musique et bruitage -l'orage, le tonnerre, les éclairs -, accompagnés d'effets visuels renforcent cette description dans l'adaptation espagnole. Il arrive de même autant pour la mort de Caesonia que pour celle de Caligula. Les angles de prises de vue, les pano travelling, les plongées, la grosseur des plans... tout contribue à donner une dimension plus efficace à la grandeur de ces images troublantes de la folie, du rythme trépident de l'action, de la tension vécue par les personnages, de la violence et de la force dramatique du héros.

Dans la proposition de l'Om Imprebìs on apprécie particulièrement le travail de réécriture théâtrale et de dramaturgie de Santiago Sánchez. Partant du respect au texte dans son aspect discursif, le metteur en scène le réécrit afin de lui apporter plus de fraîcheur et d'actualité. Pour obtenir ce résultat il fait appel à des langages non verbaux plus dans la ligne du spectacle global. Dans sa mise en scène c'est la musique de percussion qui annonce le commencement de la pièce. Le rideau où le titre de la pièce apparaissait surimprimé se lève. Une sphère énorme surgit au fond de la scène. Elle symbolise le pouvoir - pièce de monnaie dorée -, la lune - quête de l'impossible -, un œil vigilant - le pouvoir menaçant -, un miroir... Cet élément scénographique se déplacera tout au long de la représentation pour adopter des valeurs différentes selon le cas. Il sera le lieu à la manière d'un tableau où Caligula restera encadré, le miroir aussi important dans le texte source, un lit...

Quant à la première apparition de Caligula il faut dire qu'il surgit au milieu du public - un hors scène équivalant au jardin de son palais dans le texte de Camus - tandis que les autres comédiens sont installés sur le plateau suivant des mouvements chorégraphiques qui servent à traduire leur inquiétude face à l'absence de l'empereur celle dont Camus parlait au début de sa pièce (Camus, 1958:12). Cet incipit met en évidence le désir du metteur en scène d'accorder à sa représentation une dimension plus en accord avec cette idée de rupture du quatrième mur, le public, qui se voit d'une certaine manière ainsi interpelé.

14 Par rapport à l'apparence externe des acteurs il faut dire que les costumes conceptuels d'inspiration dans l'époque de l'empereur traduisent cependant une relative modernité car ils donnent au mythe du personnage et de ses sujets un caractère atemporel. Ils peuvent laisser entrevoir grâce aux fréquents changements qu'ils en font - surtout Caligula et Caesonia - le passage du temps - les actes du texte - et la relecture des scènes les plus saisissantes - danse Caligula - Venus, banquet chez Cherea, morts des héros...

15 Ces langages scéniques sont complétés par un excellent travail de la lumière. En effet l'éclairage joue un rôle décisif à l'heure de mettre l'accent sur les émotions des personnages remplaçant ce travail cinématographique de la caméra que nous avons commenté dans la version précédente. Un cyclorama changeant de tonalités et de couleurs renforce cette impression en même temps qu'il suggère des déplacements de lieux - palais, maison de Cherea, par exemple. 

salle du palais de Caligula est ici composée par un planché carré similaire aux jeux d'échecs à l'imitation du marbre, signe d'opulence et de rigueur historique. Sur lui une sépulture fourrée par une toile noire. De chaque côté, sur le sol, de grandes bougies allumées. Des fleurs. Côté jardin, le miroir ; côté cour, le gong et le maillet. Des éléments qui seront présents tout au long de la représentation. Deux marches et deux grands rideaux au fond de la scène encadrant un cyclorama vertical bleu - le ciel - où il est projeté une image de la lune. Ce tableau représente un temps antérieur au texte de Camus relatif à la mort de Drusilla. Le spectateur est plongé dans cette atmosphère funèbre qui lui signale à travers une image précise l'origine des troubles de l'empereur. Le pouvoir de Caligula est représenté par un fauteuil rouge et doré. Les sénateurs de leur côté utiliseront pour s'asseoir des cubes fourrés en noir qu'ils déplaceront à convenance. correspondance avec l'époque historique: distinction de tunique courte pour les personnages les plus jeunes ou les moins significatifs dans l'échelle sociale et de la toge prétexte pour les plus nobles et les plus âgés. Caligula et Caesonia changeront de costumes suivant les différents moments de la structure de la pièce.

Le langage rythmico-musical n'a pas été, à notre avis, très développé. La musique over, enregistrée à la manière d'une bande sonore filmique, occupera dans cette adaptation une place mineure. Elle est présente dans les monologues de Caligula et dans quelques dialogues principaux. Mais elle résulte selon notre opinion trop évidente. Le même commentaire pour l'emploi de la lumière qui reste d'un effet assez conventionnel.

\section{L'empreinte de l'acteur principal dans le rôle de Caligula11}

19 Il doit être énormément difficile pour un comédien de se libérer de la trace qu'un autre laisse associée au personnage qu'il a interprété. Réinterpréter ce personnage dont le nom seul - Caligula - impose déjà un challenge et le soustraire de manière convaincante à l'inconscient collectif des spectateurs amants du théâtre? Telle est la tâche de tous ces nouveaux « Caligula » espagnols sur la scène à l'égard de Gérard Philippe en France, de Vittorio Gasman en Italie ou de José María Rodero en Espagne.

Les adaptations que nous avons abordées jusqu'ici montrent différentes visions sur l'interprétation que ces acteurs font du personnage camusien. Chacun y laisse son empreinte. Rodero (1971) reflète pour sa part le travail d'un grand acteur consacré à la recherche de personnages immortels. Il s'investit de tout son talent pour montrer à quel point il connaît son métier issu d'une école traditionnelle où l'on donnait plus d'importance au texte d'auteur et à l'interprétation de l'acteur. Les comédiens qui l'accompagnent sur scène contribuent de par leur attitude et leur disposition sur le plateau à souligner sa présence physique et actorale tout au long de la pièce.

21 La proposition de l'Om Imprebìs (2009), offre en revanche un autre point de vue sur l'acteur principal et sur son rôle. On dirait qu'il existe une certaine tendance à le diffuser parmi les autres comédiens, à en faire un autre quelconque, un de plus. Sandro Cordero, acteur, se voit intégré par moments dans l'ensemble des comédiens de la pièce ce qui diminue sa tension et son histrionisme en faveur d'un spectacle global ${ }^{12}$. Le public ne centre pas pour autant toute son attention sur Caligula et sur l'acteur qui l'interprète

Carnets, Deuxième série - 4 | 2015 
mais par contre il a la possibilité de concevoir ce travail collectif dans sa dimension spectaculaire ${ }^{13}$.

L'adaptation de Joaquín Vida (2012) revient cependant à cette conception plus classique de l'acteur principal s'imposant sur les autres qui font partie de son jeu. En fait il y a un détail qui mérite d'être cité et qui relève de la scénographie et de l'espace occupé par les comédiens : Collados/Caligula ne quitte presque jamais le carré en marbre dessiné sur le sol - jeu d'échecs - tandis que les autres personnages le font successivement pour devenir eux-aussi des spectateurs situés sur le plateau mais à l'extérieur de ce carré symbole de l'espace du tyran. C'est à notre avis une formule qui permet de mieux focaliser l'action sur lui, de développer de manière plus efficace son travail de caractérisation du personnage.

Il est d'ailleurs également intéressant de souligner un autre aspect qui donne à cette manière de concevoir le personnage et à son interprétation une dimension et une réception $\mathrm{du}$ public bien éclectique. Nous parlons de la caractérisation externe du personnage. Il faut d'abord se rapporter à l'histoire comme référent à partir duquel sera élaboré le profil et l'aspect physique de l'empereur. Les sources parlent d'un homme laid, poilu, maigre et peu attrayant. Camus ne se soucie pas de son image extérieure si ce n'est que pour le déguiser en Venus par exemple ou pour le présenter pour la première fois sur scène lors de son retour depuis des jours d'absence. Il nous dit qu'il a un « air égaré, sale, jambes souillées » (Camus, $1958: 25)$. Les autres didascalies faisant référence à Caligula se rapportent de préférence à son comportement, à son attitude déséquilibrée, à sa logique écrasante qui laisse voir sa folie, à ses changements d'humour, à sa cruauté, à son fou rire... Les indications à ce propos constituent un véritable manuel d'interprétation à l'usage des comédiens intrépides qui oseraient aborder une telle entreprise. En effet Camus parle de : éclat soudain, avec douceur, rie et frappe, voix triomphante, air farceur, rêveur, œil mauvais, rudement, lassitude, avec colère, aimable, de façon passionnée, d'une voix faible, plus sombre que jamais, brutalement, hurlant... Toute une série de précisions qui définissent très profondément les différentes situations vécues par l'empereur et ses réactions à elles.

24 Or comment nos comédiens vivent et exécutent-ils ces notations que le texte propose ? Il faut dire que chacun possède sa technique et que les points de vue des metteurs en scène contribuent largement à remarquer des aspects individuels et particuliers selon le cas. Rodero par exemple voit renforcée son interprétation par la caméra d'Azpilicueta. Cela résulte particulièrement utile à l'heure d'offrir aux spectateurs un exercice de mimique ou de langage gestuel où les gros plans accordent plus de force aux regards, au visage... Nous apprécions de même chez lui sa brillante représentation de la folie due à la modulation et au timbre de sa voix ce qui lui permet d'insister sur les variations du rythme et de l'expression de la violence ou de la sensibilité le cas échéant. Cette forme de projection du personnage se complète par les costumes que l'acteur exhibe ajustés à l'époque qu'il représente - Rome, premières années de notre ère -, ce qui rend le personnage traditionnel en accentuant sa dimension historique. Son âge, celui du comédien, cependant, dépasse celui que Caligula avait, mais Azpilicueta équilibre de manière très lucide cette circonstance en mettant sur scène Caesonia et Cherea du même âge que lui ${ }^{14}$. À l'exception du jeune Scipion et du vieux sénateur - tous deux en accords avec leur qualification - le reste des comédiens gardent cette symétrie avec le protagoniste. 

attribuée à Caligula que le comédien exploite à volonté. D'un côté cela provient de sa manière de s'habiller, de ses vêtements moins en accord avec l'opulence de l'empereur, plus à l'usage d'une fonctionnalité demandée par la conception du spectacle. D'autre côté, son aspect général négligé, donnant l'impression de peu de souci et de grotesque - ces poils de la poitrine débordant la chemise qu'il porte, par exemple. Tous ces éléments très visuels contribuent à manifester une manière différente de concevoir et d'exprimer la folie chez lui. Ce Caligula se montrera plus naïf apparemment. Les actions sont les mêmes certes mais sa façon de parler, son rire, ses mouvements sur scène, le timbre de sa voix... tout se traduit en une vision enfantine et innocente mais brutale aussi de la méchanceté. On dirait qu'il s'amuse à jouer sur scène, que son interprétation du rôle qui lui a été assigné ainsi le lui permet et qu'il n'est pas tout à fait seul dans sa quête d'une liberté suprême telle que le personnage qu'il incarne cherche ${ }^{15}$. Un autre critère qui abonde sur cette impression vient, donné par les comédiens qui l'entourent du même âge que lui. Caesonia se montre moins mère, plus femme. Sa sensualité, sa disposition à danser, à chanter même, lui accordent plus de symétrie et d'équilibre avec le personnage de Caligula. De même pour Hélicon, jeune comédien, à un niveau similaire à son empereur de par son attitude, prêt à le suivre en tout moment, sachant danser et jouer des instruments, offrant grâce à son corps noir athlétique et vigoureux des accents très sensuels et rythmiques.

Quant à Javier Collado, il faut dire qu'il résulte le plus séduisant des tous. D'abord par son physique et puis par sa modernité grâce aux séries de la télévision où il apparaît. Cela permet de le présenter comme un personnage plus attrayant, plus capable de surprendre le spectateur hésitant entre la cruauté et l'innocence. Sa jeunesse est en rapport avec l'âge du personnage historique. De même pour Caesonia, légèrement plus âgée que lui comme dans la réalité. Par conséquent plus mère qu'épouse, plus logique sa peur de vieillir dans le texte et dans la représentation théâtrale. Cherea plus âgé que Caligula ici permettant au spectateur de croire plus facilement à la force de ses idées sur la conspiration dont il est la tête visible. Tous ces aspects contribuent en somme à remarquer le caractère vulnérable du héros qui fait souffrir son entourage mais qui se révèle de même souffrant lui aussi. D'autre part Collados ne renonce pas à s'ériger en protagoniste. Il tire profit des grands moments de son interprétation sachant qu'il est seul sur scène ou que les autres témoignent de ses qualités interprétatives. Il doit beaucoup à Rodero et à Luis Merlo bien qu'il montre moins d'affectation qu'eux.

\section{La part du spectateur. En guise de conclusion}

Le public rend actuel de par son attitude et de par sa réponse la vigueur du mythe représenté. Il accorde du sens à chaque adaptation étudiée, l'encadrant dans son contexte de réalisation, d'évocation du pouvoir exacerbé incarné par Caligula mais reconnu chez d'autres personnages historiques plus proches de lui. C'est ainsi dans ce sens d'actualité plus ou moins nuancée que les spectateurs des différentes propositions scéniques interprètent l'histoire qu'on leur présente. Ceux des années soixante-dix suivant une orientation cultivée et intellectuelle découvrent un texte et une représention théâtrale de la folie et de la tyrannie que la censure n'a pas su interpréter dans toute sa dimension de contenu et de temporalité. La perspective historique de l'œuvre de Camus est dépassée par les réflexions de ses personnages. Sur la scène espagnole on dirait que ce point de vue 
a diminué la violence des actes de l'empereur ainsi que les références explicites à l'inceste, à sa vie dépravée, à la vengeance extrême et au mépris de la religion et des valeurs de la société.

Suivant cette appréciation nous pouvons affirmer qu'Azpilicueta reste par conséquent fidèle aux principes élaborés au niveau du tissu textuel et des images offertes par Camus, conscient que son public - éminemment cultivé - saura comprendre qu'on lui offre sans souci de bienséance les meurtres sur scènes tandis qu'on lui épargne les élans érotiques de l'empereur qui seront réservés pour le hors-scène.

Bien différent du pari de Santiago Sánchez. Il pense à son public du XXI ${ }^{e}$ siècle. Public de l'image, du regard sans tabous. Cela fait qu'il manifeste plus d'intérêt à ces autres langages esthétiques beaucoup plus explicites que la parole. Un bon exemple, la scène chez Cherea, représentant le banquet comme si c'était la Sainte Cène, mais ayant les différents personnages éparpillés de manière grossière par terre. Faute de bancs ou de sièges pour s'asseoir ils seront installés directement sur le sol ou sur des gradins ce qui se traduit par une sensation de sensualité et de bacchanale orgiastique. D'autre part l'attitude de Caligula à l'égard de Caesonia, leurs échanges érotico-sensuels en public, mettent en évidence ce désir de rendre plus logique la scène et d'attirer le regard complice et participatif du spectateur-voyeur.

Même intérêt à l'insu de Joaquín Vida. Son adaptation manifeste un des moments les plus sordides et les plus scabreux : le viol de la femme de Mucius se produit sur scène devant tous les autres personnages parmi lesquels le mari lui-même. Cette cruauté met en relief le caractère dépravé de l'empereur et justifie de manière plus éloquente le dégoût des autres personnages, le désir de vengeance du mari outragé et la douleur - plus humaine et plus logique - de Caesonia ainsi outragée. Le public pour sa part est saisi d'horreur mais il faut convenir que son interprétation de la scène obéit à une réaction absolument réaliste de la même qui ne relève pas de la pertinence ou non de l'exhiber sur scène.

Cela dit il faut souligner en guise de conclusion qu'un metteur en scène compétent et soucieux de son destinataire et du sens du spectacle théâtral, assisté d'un acteur à forte empreinte et d'un texte immortel, peuvent séduire les publics les plus exigeants et les plus éclectiques. L'essentiel c'est d'identifier ce spectateur auquel on s'adresse et de décider si l'on prétend garantir le succès de la réception ou si, en revanche, on préfère se maintenir fidèle à un style personnel préconçu et de défier ainsi l'univers d'attente de notre spectateur actuel.

\section{BIBLIOGRAPHIE}

AZPILICUETA, Jaime. (1971). [disponible le 16/03/2011] <URL: http://www.rtve.es/alacarta/videos/ estudio-1/estudio-1-caligula/861793>.

BONHOMMmE, Béatrice (1996). Le roman au XXe siècle à travers dix auteurs de Proust au Nouveau Roman. Paris : Ellipses. 
CAMUS, Albert (1958). Le malentendu, suivi de Caligula, édition numérique réalisée par Jean-Marie Tremblay, bénévole, professeur de sociologie au Cégep de Chicoutimi et fondateur des Classiques des Sciences Sociales, à partir d'Albert Camus [1913-1960] (1944). Caligula, pièce en quatre actes.

EUROPAPRESS. lainformacion.com [disponible le 13/12/2013] <URL : http://

noticias.lainformacion.com/arte-cultura-y-espectaculos/teatro/el-gran-teatro-acoge-este-

viernes-caligula-de-camus-que-indaga-en-el-absurdo-de-la-

existencia_TLOZNjGwy1sRY6psZAQhh3>.

FRANCHESCINI, Paul-Jean et LUNEL, Pierre (2011). Caligula. Barcelona : Zeta.

IMPREBÌs [disponible le 21/10/2014] <URL : http://www.imprebis.com/espectaculos.asp?

idContenido $=7>$.

INAEM 4936. Calígula. Autor : Camus, Albert. Versión : Sánchez, Santiago. L'Om-Imprebís, Teatro

Fernán Gómez, de Madrid 07/04/2010.

KRISTEVA, Julia (1988). Étrangers à nous-mêmes. Paris : Librairie Arthème Fayard.

MOLINA, Margot. [disponible le 08/09/2013] <URL : http://elpais.com/diario/2009/09/09/

andalucia/1252448534_850215.html>.

MUÑOz, José Antonio. [disponible le 17 /01/2013] <URL : http://www.ideal.es/granada/

$\mathrm{v} / 20130117 /$ cultura/caligula-dice-cara-cosas-20130117.html>.

PINEL, Vincent (1996). Vocabulaire technique du cinéma. Paris : Nathan Université.

\section{NOTES}

1. Cette liberté que seule la mort rend possible. Ou comme souligne Béatrice Bonhomme « l'homme vraiment libre et celui qui, acceptant la mort comme telle, en accepte du même coup les conséquences, c'est-à-dire le renversement de toutes les valeurs traditionnelles de la vie » (Bonhomme, $1996: 48$ ).

2. Le cinéma a tendance à aborder le personnage de Caligula d'une manière très frivole restant à la surface d'un stéréotype de la dépravation sexuelle et du sadisme. Il y a cependant des réalisations qui méritent d'être retenues, telles que le film de Tinto Brass (1979), avec la participation de Peter O'Toole et de Hellen Mirrem, considéré un drame érotique. En revanche le théâtre accorde à l'étude du personnage une autre dimension. Les mises en scène en France et à l'étranger mettent l'accent sur la maladie de l'empereur et sur son déséquilibre. Elles offrent aussi des perspectives nouvelles sur l'art scénique et sur l'interprétation d'un mythe historique tel que Caligula l'est maintenant. Citons les propositions de Valérie Fruaut (2009) qui propose une mise en scène moderne, basée sur un décor composé par des chaises transparentes et un fauteuil blanc, de grandes tables noires, enveloppé l'ensemble par une musique intrigante, une lumière bleue et blanche assez froide et des costumes où la cravate remplace la toge romaine sans pour autant diminuer l'effet personnage d'une élite sociale ; celle de Stéphane Olivié-Bisson (2011) où règne l'ambigüité, la complexité de la nature humaine; ou celle de Patrick Rouzaud (2013), qui situe le dilemme du personnage camusien au centre de sa quête provocant l'amour et la haine d'un personnage qui passe souvent de bourreau à victime et vice-versa, inondant la scène de son désir véhément de liberté. Pour la littérature, nous avons eu l'opportunité de lire récemment le roman écrit par Franchescini et Lunel, créé à partir de diverses sources historiques donnant à la personnalité du tyran des traits aussi cruels que fascinants. (Franchescini et Lunel, 2011). 
3. Tamayo a présenté cette même pièce plus tard au même endroit. C'était en 1990 et l'acteur principal cette fois-ci était Imanol Arias, icône de la séduction masculine à l'écran cinématélévision.

4. Voir références bibliographiques pour le texte de Camus qui fera l'objet de toutes nos citations.

5. Nous sommes conscients de la diversité de propositions scéniques existantes et de leur qualité, mais nous avons voulu restreindre le corpus de notre analyse à ces trois uniquement afin de donner plus de profondeur à notre étude. Cela ne nous empêche pas de mentionner au moins à part celle de Tamayo-Imanol Arias déjà citée plus haut, celle de Tamayo-Luis Merlo (1995), celle de Oskar Salcedo présentée lors des Jornadas Romanas de Valeria Condita (2008), celle de Eloy de la Iglesia-Roger Pera, opéra prima en théâtre du cinéaste dont la critique éloge l'enregistrement en plein air et les effets audiovisuels, parmi d'autres qui offrent autant de possibilités scéniques à partir de la même source.

6. Ils ont élaboré à ce propos un excellent dossier à contenu publicitaire et éducatif de leur pièce qui nous a fourni un matériel très pertinent pour nos recherches (Imprebìs, 2013).

7. Cosmoarte souligne qu'il s'agit de «la primera versión completa que se estrena, porque en España se han hecho montajes con actores de primera fila, como José María Rodero, Imanol Arias o Luis Merlo; por cierto, con otro granadino, José Tamayo, al frente. A nuestro paisano aquel estreno le trajo muchos quebraderos de cabeza, porque se hizo viviendo Franco, en 1962 o 1963, y con la censura en pleno apogeo. Por eso, el montaje que se estrena este fin de semana en Granada es una buena oportunidad para ver la obra tal como Camus la escribió » (Muñoz, 2013).

8. Nous allons nous occuper des aspects les plus significatifs des langages non verbaux qui sont présents dans les trois versions objet de notre recherche et qui ont comme point de départ le texte source de Camus. Dans ce sens l'espace référentiel et scénique, le hors-champs, les costumes, la lumière et la musique ainsi que le bruitage seront les éléments les plus cités car c'est bien à travers ceux-ci que les adaptations de la pièce de Camus témoignent de l'actualité qu'elles proposent.

9. Pour les références aux mots techniques concernant les langages filmiques nous nous sommes servis du livre de Vincent Pinel (Pinel, 1996).

10. À souligner par exemple la reproduction à travers la musique des bruits d'armes que Caligula mentionne dans la scène finale précédant son massacre aux mains des sénateurs et de Cherea (Camus, $1958: 206$ ).

11. Imanol Arias parlait en 1990 de la difficulté pour un acteur de jouer ce rôle, un commentaire que la plupart des acteurs qui l'ont abordé partagent. "Sandro Cordero, que encarna al joven e impulsivo emperador Calígula, confesó ayer que estaba 'aterrado' ante el reto » (Molina, 2009: sans p.).

12. La scène de la danse de Caligula travesti en déesse Vénus est exécutée par tous les comédienspersonnages qui bougent et chantent de façon frénétique inspirés par des rythmes africains très sensuels. Caligula acteur quitte de temps en temps son rôle pour devenir joueur de tambour à côté des autres musiciens.

13. «Gracias al planteamiento de la compañía, en la que todos trabajamos en la misma dirección, el terror ha desaparecido y ahora me siento muy arropado sobre el escenario » (Molina, 2009: sans p.).

14. Cette situation explique que Caesonia puisse s'exercer comme mère et comme épouse en même temps sans choquer le spectateur. Elle est l'épouse complaisante qui mime les gestes et les comportements de son amour. Elle sera aussi la femme protectrice qui l'accueillera dans son sein aux moments où il manifeste plus de faiblesse. Cherea, pour sa part, représente de manière plausible sa rivalité et sa raison d'être face aux caprices inouïs de l'empereur. 
15. «L'absolu de cette liberté s'appelle pourtant solitude », (Kristeva, 1988 : 23). Les personnages interprétés par les autres acteurs sont noyés de manière flagrante dans cette solitude à double voie : celle de l'acteur et celle du rôle incarné par eux.

\section{RÉSUMÉS}

Nous avons voulu rendre hommage à Albert Camus dès l'Espagne théâtrale récente. Nous avons choisi pour notre objectif une de ses pièces les plus réinterprétées sur les tréteaux espagnols : Caligula. Nous dévoilerons les clés de relecture de certains metteurs en scène du texte de Camus, le transcodage du littéraire au spectaculaire et l'adaptation culturelle et temporelle dont la pièce a fait l'objet.

In this paper we aim to pay tribute to Albert Camus by studying its presence in the most recent Spanish drama. For our purposes, we have chosen Caligula, one of his pieces which have been reinterpreted more often by Spanish playwrights. We will unveil the keys of the reiterpretation of this text by some directors; we will also observe the way the literary text is transformed into a theatrical even; finally, we will pay attention to the issues of its cultural and temporal adaptation.

\section{INDEX}

Mots-clés : littérature dramatique, spectacle théâtral, transcodage, adaptation culturelle, comparatisme

Keywords : dramatic literature, theatrical performance, transcoding, cultural adaptation, comparativism

\section{AUTEUR}

\section{RAFAEL RUIZ ÁLVAREZ}

Un. de Grenade

rruizal[at]ugr.es 